\title{
Zeb1 is important for proper cleavage plane orientation of dividing progenitors and neuronal migration in the mouse neocortex
}

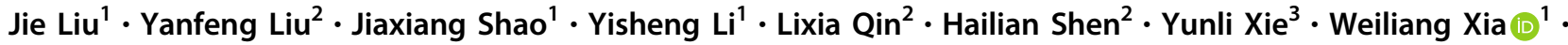 \\ Wei-Qiang Gao ${ }^{1,2}$
}

Received: 8 May 2018 / Revised: 4 November 2018 / Accepted: 4 December 2018 / Published online: 11 March 2019

(c) ADMC Associazione Differenziamento e Morte Cellulare 2019

\begin{abstract}
During neocortical development, there are two important events, including expansion of the neural progenitor pool through symmetric divisions, and generation of neurons via asymmetrical divisions that lead to a serial process of neuronal polarization, migration, and layer-type specific phenotype acquisition. The mechanisms underlying these processes remain poorly elucidated. Here, we show that the transcription factor Zeb1 regulates the orientation of the cleavage plane of dividing neural progenitors, neuronal polarity, and migration. Upon Zeb1 removal, the cleavage plane of mitotic neural progenitors fails to orientate vertically, resulting in random orientation and premature neuronal differentiation. Consequently, these extra number of precociously produced neurons migrate aberrantly to the upper layer. Mechanistically, we show that Zeb1 suppresses Pak3, a p21-activated serine/threonine protein kinase, through formation of a functional repressing complex together with methyltransferase PRMT5 and Pak3. Our results reveal that Zeb1 plays an essential role in neocortical development and may provide insights into the mechanisms responsible for cortical developmental diseases.
\end{abstract}

\section{Introduction}

The mammalian cortex consists of a six-layered laminar of neurons in a highly organized fashion [1,2]. The principal subtype of progenitors that produce most cortical neurons in the neocortex is the radial glial cells (RGCs), stemming

Edited by N. Bazan

Supplementary information The online version of this article (https:// doi.org/10.1038/s41418-019-0314-9) contains supplementary material, which is available to authorized users.

Weiliang Xia

wlxia@sjtu.edu.cn

Wei-Qiang Gao

gao.weiqiang@sjtu.edu.cn

1 State Key Laboratory of Oncogenes and Related Genes, Ren Ji Hospital, School of Biomedical Engineering \& Med-X Research Institute, Shanghai Jiao Tong University, Shanghai 200030, China

2 Renji-Med X Clinical Stem Cell Research Center, Ren Ji Hospital, School of Medicine, Shanghai Jiao Tong University, Shanghai 200127, China

3 State Key Laboratory of Medical Neurobiology \& Institutes of Brain Science, Fudan University, Shanghai 200032, China from neuroepithelial cells located in the ventricular zone $[3,4]$. At the onset of neurogenesis (at E9-E13), RGCs divide symmetrically, which produce two daughter cells that retain RGC property to expand the progenitor pool, or asymmetrically, which give rise to either one neuron and one RGC or produce one basal progenitor (BP, also called intermediate progenitor) and one RGC at E13-E18 [5-7]. The newly born post-mitotic neurons migrate from their original site to their final destination [8]. Defects in this process result in various brain disorders [9-11]. To date, how apical RGCs precisely self-renew versus differentiate and what intracellular machinery endows newly generated neurons a motile behavior to form the intricate cortical structures are still poorly elucidated.

During neural development, epithelial cells delaminate from the epithelial sheet and acquire the mesenchymal phenotype [12-14]. Many intracellular molecules participate in this epithelial mesenchymal transition (EMT) process. Among them, Zeb1 is a potent one [15]. For example, it has been shown that in several tissues, Zeb1 can repress transcriptional activities as it preferentially binds to the CACCT sequence of the target genes [16-18]. However, whether Zeb1 affects cortical neurogenesis and neuronal migration and whether it has new downstream effectors have not been determined. 
In this study, we provide evidence that Zeb1 is an essential factor for neocortical development. Deficiency of Zeb1 promoted RGCs' exit from cell cycles and their precocious differentiation into neurons via affecting the cleavage plane orientation, resulting in the premature production of post-mitotic neurons and their aberrant migration toward their final destination. In addition, we identified Pak3, a p21-activated serine/threonine protein kinase, as a direct Zeb1 effector that controls cortical neuronal polarization and migration.

\section{Results}

\section{Zeb1 is exclusively expressed in RGCs in the developing cortex}

To assess the role of Zeb1 during neocortical development, we first examined its expression pattern in the developing cortex. Western blot analysis of cortical lysates revealed that Zeb1 expression was initially detected at E9.5 when the thin cortex is mainly composed of progenitors, persisted until the end of neurogenesis, and then was downregulated at the postnatal stage (Fig. 1a), suggesting that Zeb1 is expressed during active neocortical neurogenesis.

Next, we performed immunostaining on cryosections of mouse brains E15.5 and found that Zeb1 was robustly expressed in Nestin- and Sox2-positive neural progenitors residing in the ventricular zone (VZ)/subventricular zone (SVZ) (Fig. 1b). Notably, Zeb1 immunofluorescence was strong at the VZ, where RGCs reside. There was an overlapping staining of Zeb1 with the RGC marker Pax6, but rarely with the basal progenitor cell (BP) marker Tbr2 (Fig. 1c). Consistently, Zeb1 was also expressed in nuclei of cultured neurospheres generated from neural progenitors prepared from the E13.5 cortex (Fig. 1d). Conversely, Zeb1 was hardly detected in the doublecortin (DCX)-positive immature neurons (Fig. 1e), which represent postmitotic, early differentiated neurons.

\section{Zeb1 is essential for cortical layer formation}

The selective expression pattern of Zeb1 in RGCs suggested a potential role of Zeb1 in neurogenesis. We next studied Zeb1 knockout mouse phenotypes [16]. Immunostaining was performed to confirm the knockout efficiency of Zeb1 at E15.5 in Zeb1 knockout mice compared with wild-type littermates (Fig. S1). Zeb1 knockout mice died shortly after birth. As Zeb1 was highly expressed in RGCs, we asked whether depletion of Zeb1 led to neurogenesis defects. We first examined whether the laminar architecture of cortex is affected by Zeb1 ablation. Immunostaining revealed that the cortical layers of E18.5 Zeb1 knockout brains showed aberrant patterns as compared with their wild-type counterparts. Notably, a considerable number of Brn2-positive neurons, which are born during late neurogenesis (from E14.5-E18.5), were produced and located not only in the upper layers, but also in deep layers. As such, these Brn2positive neurons were plausibly found to be on their way to layer II-III, raising the possibility that Zeb1 exerted a function during the production and influenced the migration of later-born neurons (Fig. 2a, b). Compared with the laterborn Brn2-positive neurons, the number of early-born Ctip2-expressing deep-layer neurons (E10.5-E14.5) was not significantly changed (Fig. 2c, d). However, the number of Tbr1-expressing deep-layer neurons was decreased (Fig. 2e, f). Thus, Zeb1 is required for the proper differentiation and migration of post-mitotic neurons and the appropriate cortical layer formation during neurogenesis.

\section{Zeb1 is required for the maintenance of the progenitor pool at the onset of neurogenesis}

To explore whether Zeb1 affects progenitor proliferation in the neocortex, we stained wild-type and Zeb1 knockout mice brain coronal sections for phosphor-Histone H3 (PH3) to label mitotic progenitors. At E13.5, the numbers of PH3positive progenitors in wild-type and knockout brains were comparable. However, at E14.5, the number of mitotic progenitors was notably decreased in the knockout group (Fig. 3a, d). Next, we examined the number of progenitors in the Zeb1 knockout mice, by examination of Pax6positive RGCs and Tbr2-positive BPs. We found that Pax6positive RGCs were depleted and the number per unit was significantly reduced compared with the wild-type group in the absence of Zeb1 at the stage of E15.5 (Fig. 3b, e). Reversely, the number of Tbr2-positive BPs per unit, destined to become neurons, was dramatically increased in the VZ/SVZ of Zeb1 knockout mice than that of the wild-type group (Fig. 3b, f). Furthermore, we found that knockout of Zeb1 did not affect RGCs polarity relative to the apical side of neuroepithelium, as revealed by immunostaining for Nestin and adherens junctions ZO-1 (Fig. S3).

To gain further insights into how Zeb1 knockout could affect the progenitor pool size, we examined the proliferative status of $\mathrm{VZ}$ progenitors by performing EdU pulse labeling in combination with Ki67 staining, to visualize Sphase cells and reveal cells within the cell cycle. We labeled cycling progenitors by EdU incorporation at E14.5 and stained for Ki67, a proliferation marker, $24 \mathrm{~h}$ later, at E15.5, and then we quantified cells that exit from the cell cycle $\left(\mathrm{EdU}^{+} \mathrm{Ki}^{-} 7^{-}\right.$) (Fig. 3c, g). The proportion of EdU-positive and Ki67-negative cells was elevated in the Zeb1 knockout group. Immunostaining for cleaved caspase-3 at E15.5 revealed that cell survival was unaffected (Fig. S2), excluding the possibility that decrease in the number of 
Fig. 1 Distribution and localization of Zeb1 in the developing mouse cortex. a Zeb1 levels in cortical lysates at different developmental stages. $\beta$-actin was used as a loading control. b Coronal cryosections of the neocortex at E15.5 immunostained for Zeb1 (green) and neural stem cell marker Nestin (upper panel) and Sox2 (lower panel). c Zeb1

colocalized with radial glial cell (RGC) marker Pax6 (upper panel), but not with basal progenitor cell (BP) marker Tbr2 (lower panel). Section was counterstained with DAPI (blue). d Zeb1 is expressed in neurospheres cultured in vitro. Primary progenitor cells were isolated from the E13.5 cortex and cultured into neurospheres 2 days later.

Immunocytochemical analysis was performed on the neurospheres using antibodies against Zeb1 (green) and Sox2 (red) (upper panel) and Nestin (red) (lower panel). The nuclei were stained using DAPI (blue). e Zeb1 (green) did not colocalize with Doublecortin (red). Section was counterstained with DAPI (blue). Scale bars represent 25 $\mu \mathrm{m}$ in $(\mathbf{b})$ and $(\mathbf{c}), 50 \mu \mathrm{m}$ in $(\mathbf{d}, \mathbf{e})$

a

\section{E9.5 E11.5 E13.5 E15.5 E17.5 P2}

\section{Zeb1}
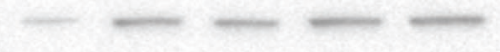
$210 \mathrm{k}$ Da

actin
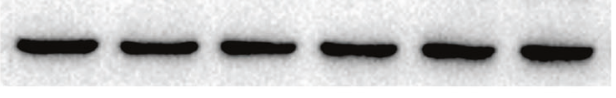

$42 \mathrm{kDa}$

b

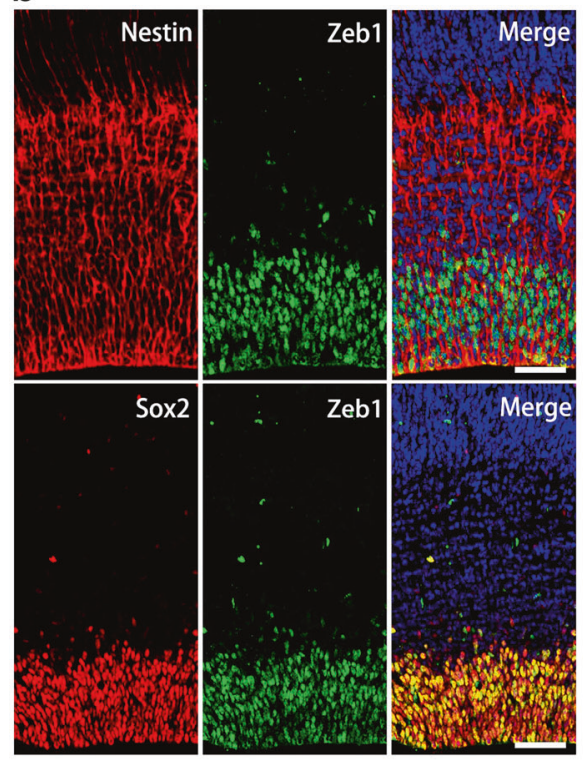

d

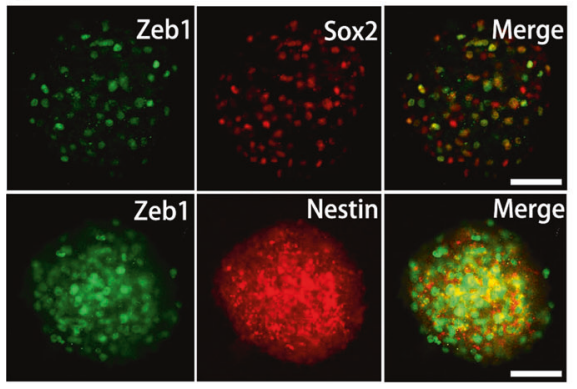

C

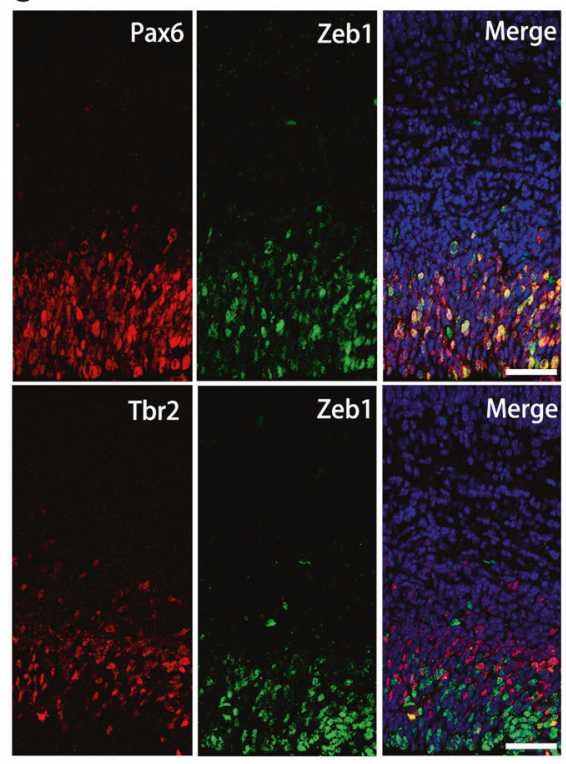

e

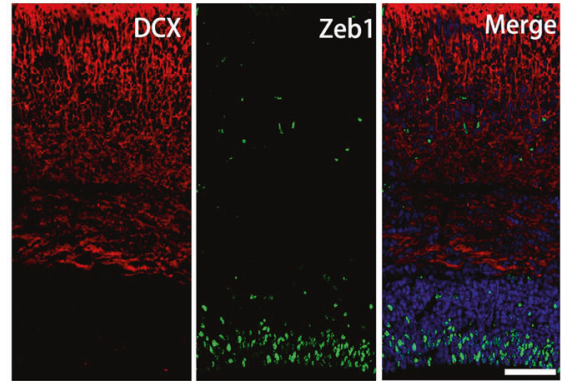

proliferating progenitors was due to apoptosis. Therefore, Zeb1 is necessary for the maintenance of the progenitor pool, preventing from premature neuronal differentiation during cortical development.

\section{Zeb1 is essential for proper cleavage-plane orientation in dividing RGCs}

During neurogenesis, RGCs undergo symmetric proliferative divisions to expand the pool of progenitors [8]. As we observed that Zeb1 knockout favored progenitor differentiation and cell-cycle exit, one potential function of Zeb1 in modulating progenitor differentiation is to promote symmetric cell division of RGCs. To verify this possibility, we measured the orientation of the cleavage plane that is correlated with the fate of daughter cells in dividing RGCs at VZ/SVZ in Zeb1 knockout mice (Fig. 3h). Because the metaphase plate of dividing progenitors in the cortex is not set until the anaphase, we focused on progenitors at the anaphase/telophase, as visualized by chromosomal arrangement (DAPI) and P-Vimentin staining, which specifically recognizes dividing progenitor cells in the developing neocortex and reveals their morphology [19]. We analyzed the cleavage-plane orientation on the basis of three-dimensional analysis that divided the angles of cleavage plane into the vertical $\left(60^{\circ}-90^{\circ}\right)$, oblique $\left(30^{\circ}-60^{\circ}\right)$, and horizontal $\left(0^{\circ}-30^{\circ}\right)$ groups. We found that vertical division was more frequent in the wild-type group (78.6\%) than in the Zeb1 knockout group (46.0\%). Conversely, the frequency of oblique and horizontal divisions increased in 
Fig. 2 Zeb1 is essential for the cortical lamination in the developing mouse brain. a, c, e Confocal images of E18.5 coronal sections from wild-type (WT) and Zeb1 knockout (KO) stained with layer-specific marker Brn2 (layer II-III), Ctip2 (IV), and Tbr1 (layer V-VI) in green. Sections were counterstained with DAPI (blue). b, d, $\mathbf{f}$ The number of $\mathrm{Brn}^{+}$, Ctip2 ${ }^{+}$, and $\mathrm{Tbr}^{+}$cells were measured in $150 \mu \mathrm{m}(n=$ 4-5 embryos, $n=6-10$ sections per group). Error bars represent SEM. $* p<0.05$, Student's $t$ test. Scale bars represent $50 \mu \mathrm{m}$

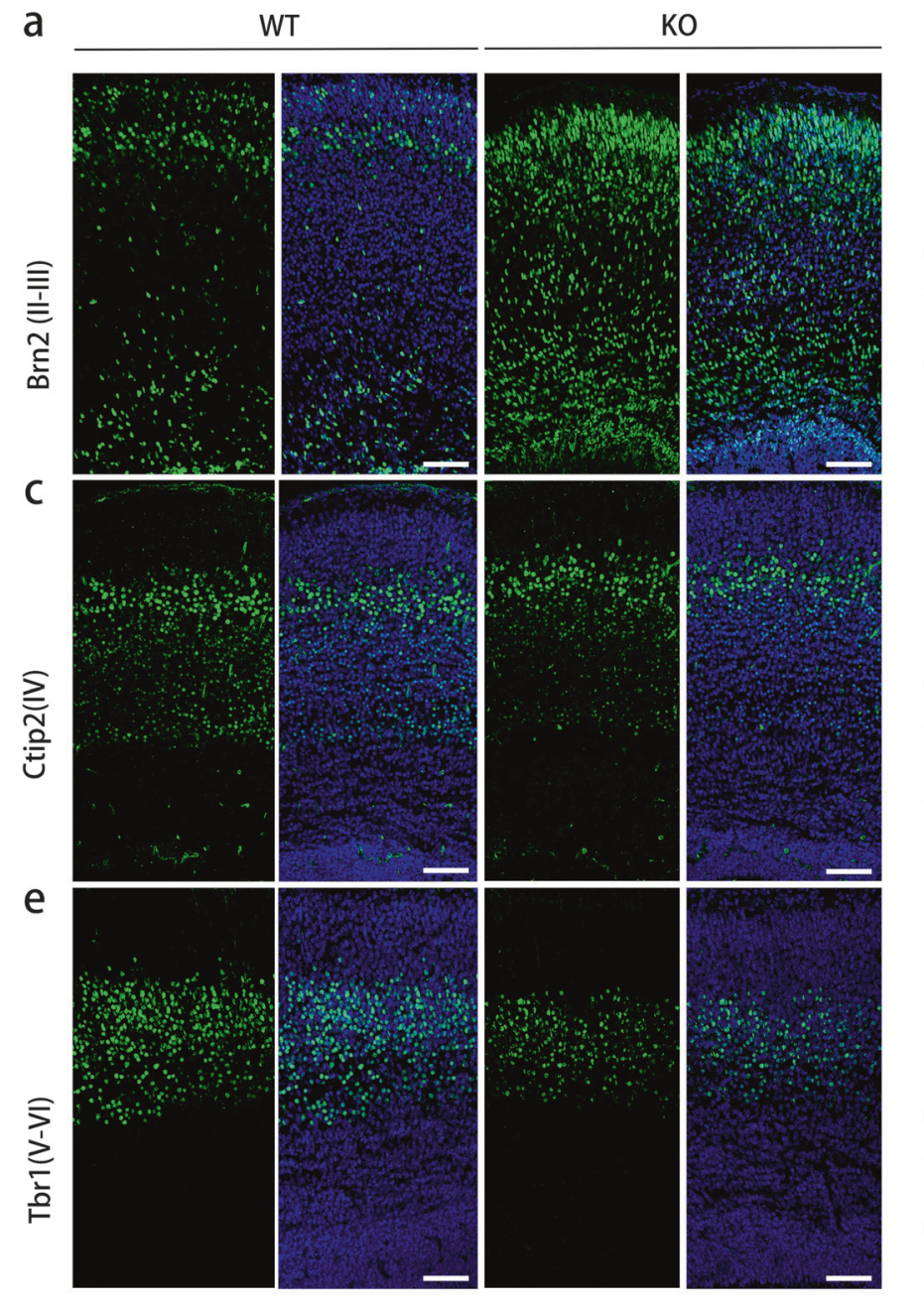

b
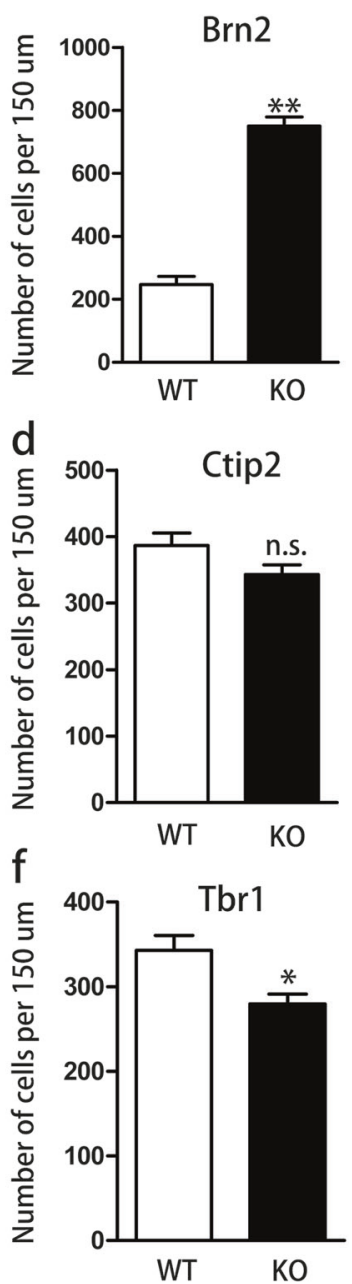

the Zeb1 knockout group (oblique 26\%, horizontal 28\%) compared with the wild-type group (oblique 19.7\%, horizontal 1.7\%) (Fig. 3i). These results suggest that Zeb1 is essential for proper cleavage-plane orientation in dividing RGCs, which regulates neurogenesis of RGCs.

To further corroborate our in vivo findings, we then asked whether Zeb1 could also affect the mode of cell division of RGCs in vitro. To assay this, we performed the pair cell analysis that was used to trace the fate of daughter cells, using markers for progenitors (Pax6) and neurons (Tuj1) (Fig. 3j, k). As shown above in Fig. 1b-d, Zeb1 was highly colocalized with $\mathrm{Sox}^{+}$and $\mathrm{Pax}^{+}$cells, hence dissociated progenitors from the anterior cortex of E14.5 should mostly consist of Zeb $1^{+}$progenitors. As such, progenitors were directly plated at clonal density and allowed to undergo one cell division in vitro. Pairs of cells generated by the division of progenitors were fixed $24 \mathrm{~h}$ after plating and analyzed by staining with the Pax 6 and Tuj1 antibodies. Progenitor cells exhibit three modes of cell division: P-P division, two daughter progenitor cells (Pax6 positive); $\mathrm{P}-\mathrm{N}$ division, one daughter progenitor cell (Pax6 positive) and one daughter neuron (Tuj1 positive); $\mathrm{N}-\mathrm{N}$ division, two daughter neurons (Tuj1 positive). Consistent with previous studies [20], the majority of RGCs derived from wild-type cortex divided symmetrically; however, the proportion of RGCs derived from Zeb1 knockout mice that divided asymmetrically were dramatically increased, indicating that Zeb1 is essential for the proper cleavage-plane orientation of RGCs.

\section{Deficiency of Zeb1 promotes migration of late-born neurons}

The mammalian cortex consists of an extraordinary number of neurons that are essential for higher-order brain functions $[21,22]$. As we observed, a considerable number of Brn2positive neurons (later-born neurons) in the Zeb1 knockout group resided in layer II-III and are much closer to the 
a

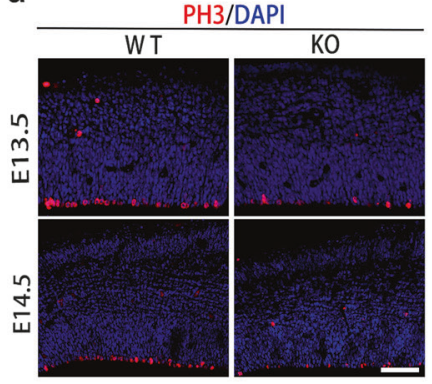

C

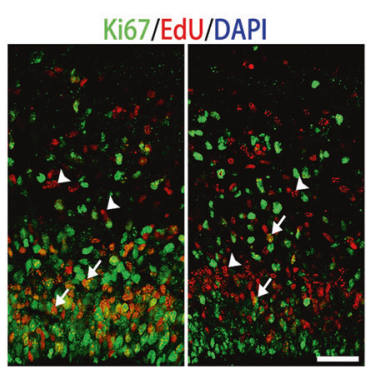

h

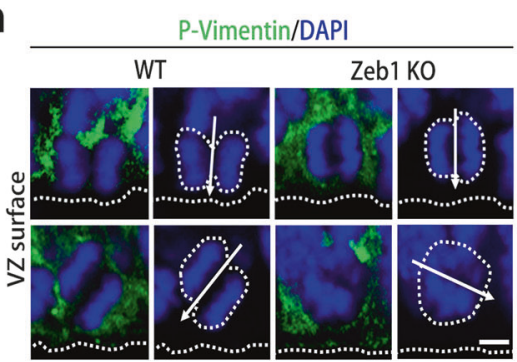

j

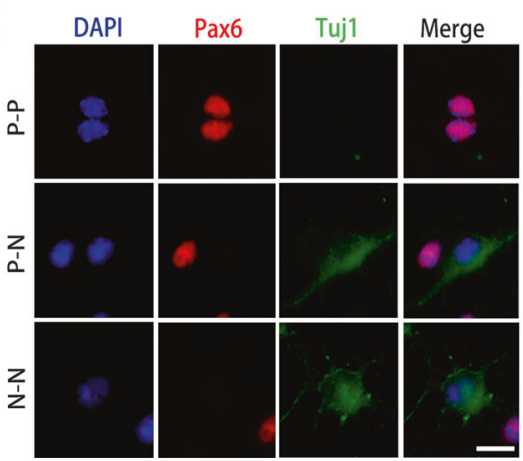

b
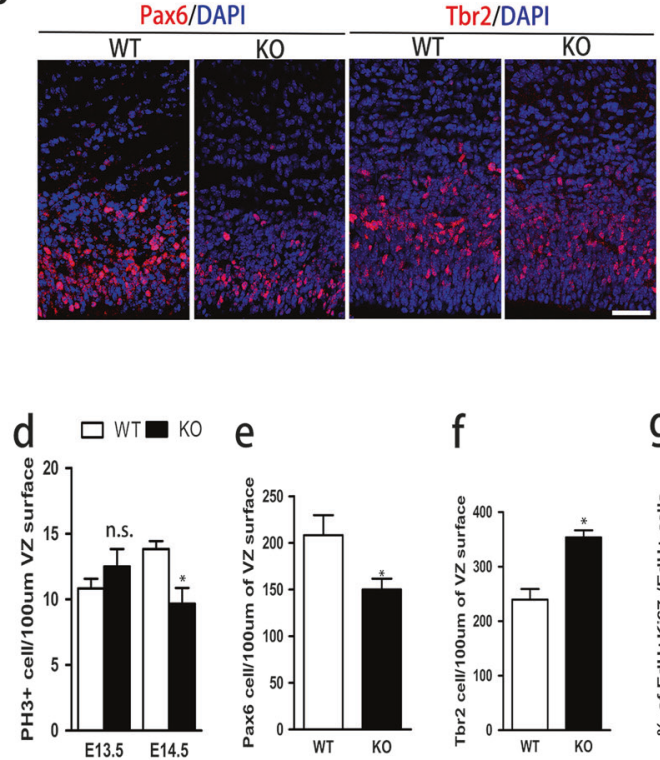

f
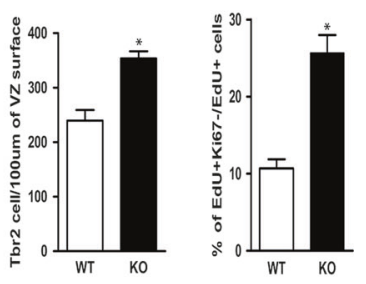

i

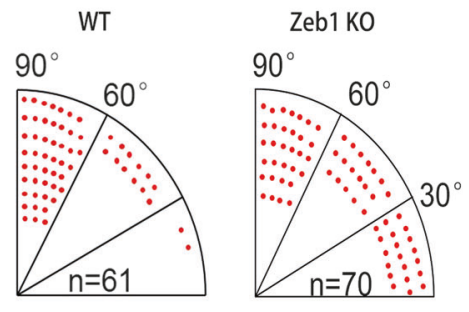

k

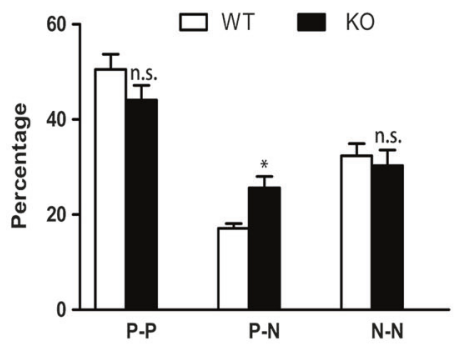

introduced the more effective shZeb1-2, followed by an IRES-GFP cassette into E14.5 mouse brains by in utero electroporation (IUE) (Fig. 4). Electroporated brains were assessed at sequential time points from E16.5, when electroporated migratory neurons left the progenitor zone entering the SVZ/IZ to P2, when most cells reached their final sites. At E16.5, 2 days after electroporation, significantly more GFP-positive Zeb1 knockdown cells were seen to migrate away from VZ/SVZ, became located in the IZ (Fig. 4a, e). Furthermore, at E17.5 and E18.5, 3 and 
Fig. 3 Zeb1 modulates the VZ progenitor pool size and the orientation of the cleavage plane of neural progenitors. a Confocal images of coronal sections from wild-type (WT) and Zeb1 knockout (KO) at indicated developmental stages were stained for PH3 and DAPI. b RGCs and BPs were identified by staining for Pax6 and Tbr2, respectively, in WT and Zeb1 KO. c Analysis of cell-cycle exit. Mouse embryos were sectioned at E15.5 and immunostained for Edu and Ki67. Arrow, cycling $\mathrm{Ki}^{+} 7^{+} \mathrm{Edu}^{+}$cells; arrowhead, $\mathrm{Ki}^{-} 7^{-} \mathrm{Edu}^{+}$cells withdrawn from the cell cycle. d-g Quantification of $\mathrm{PH}^{+}, \mathrm{Pax}^{+}$, $\mathrm{Tbr} 2^{+}$cell numbers and $\mathrm{Ki}^{-} 7^{-} \mathrm{Edu}^{+}$cells number. $\mathbf{h}$ Representative image of mitotic cells labeled by P-Vimentin (green) in the anaphase/ telophase revealed by DAPI staining (blue) at the VZ surface in E15.5 WT and Zeb1 KO cortices. Broken lines indicate the contours of dividing cells and ventricular surface. Arrow indicates the cleavage plane. Determination of the cleavage-plane orientation as the angle between the cleavage (arrows) and the VZ surface is shown on the right of image. i Each red dot represents one dividing cell (WT, 61 cells from three experiments; Zeb1 KO, 70 cells from three experiments). Data are shown as mean \pm SEM. ${ }^{*} p<0.05$. j Representative images of three different types of daughter cell pairs originating from individual radial glial cells (RGCs): (P-P) two RGCs, (P$\mathrm{N})$ one RGC and one post-mitotic neuron, $(\mathrm{N}-\mathrm{N})$ two post-mitotic neurons. Sibling cells pair radial glial cells were immunostained with the antibodies against Pax6 (red), a RGC marker, and Tuj1 (green), a neuronal marker, and counterstained DNA with DAPI (blue). k Quantification of the percentage of $\mathrm{P}-\mathrm{P}, \mathrm{P}-\mathrm{N}$, and $\mathrm{N}-\mathrm{N}$ daughter cell pairs derived from WT RGCs or Zeb1 KO RGCs (WT, 512 cells, from four experiments; Zeb1 KO, 438 cells from five experiments) Data are shown as mean \pm SEM. ${ }^{*} p<0.05$. Scale bars represent $50 \mu \mathrm{m}$ in (a), $20 \mu \mathrm{m}$ in $(\mathbf{b}, \mathbf{c}), 5 \mu \mathrm{m}$ in $(\mathbf{h})$, and $10 \mu \mathrm{m}$ in $(\mathbf{j})$

4 days after electroporation, many more GFP-positive Zeb1 knockdown cells were found to arrive in the CP compared with controls (Fig. 4b, c, f, g). At P2, no significant difference was found in the control and Zeb1 knockdown group (Fig. 4d, h). To further analyze neuronal distribution, we divided the neocortex into five parts (from bin 1 to bin 5). As observed, the positions of Zeb1 knockdown cells were similar to the control group in bin 1 (Fig. $4 \mathrm{~d}, \mathrm{~h}$ ), implicating that later-born neurons in the deep-layer cortex of Zeb1 knockout mice might have migrated to their destined final position in layer II-III at times later than E18.5.

\section{Pak3 acts as a downstream target of Zeb1 to control radial migration and multipolar-bipolar transition of cortical neurons}

To determine candidate targets acting downstream of Zeb1, we performed a pilot microarray-based gene expression profiling using progenitor cells collected from E14.5 cortical tissues of control littermates and Zeb1 knockout mice. Microarray data were confirmed by qRT-PCR and based on these analyses. Pak3 was dramatically upregulated in the Zeb1 null group. A previous report suggested that Pak3 promotes neuronal differentiation and migration in Xenopus [23]. Therefore, we examined the potential relationship between Zeb1 and Pak3 in neocortex development.
Quantitative real-time PCR analysis confirmed three- to fourfold upregulation of Pak3 at E13.5 and E15.5 in vivo (Fig. 5a) and a gradual upregulation along with progenitor differentiation in vitro (data not shown). Using UCSC Genome Browser, we identified two putative regulatory sites that contain CACCTG and CACTTG in the Pak3 promoter, which are highly conservative with the Zeb1 canonical binding sequence CACCTG [16]. To test whether Zeb1 functioned as a modulator of Pak3, we performed the luciferase assay, indicating that Zeb1 negatively modulated the Pak3 luciferase reporter driven by the $2 \mathrm{~kb}$ Pak3 regulatory element (Fig. 5b). Together, these data indicated this element performs functional repression through binding to Zeb1.

To further determine whether Zeb1 indeed exerts its function through binding these two sites, we constructed luciferase reporter containing site $\mathrm{A}$, site $\mathrm{B}$, and negative control fragment $\mathrm{C}$, respectively. Overexpression of Zeb1 downregulated the luciferase reporter activity driven by site $\mathrm{A}$ and site $\mathrm{B}$, but not the negative control fragment $\mathrm{C}$. Conversely, knockdown of Zeb1 enhanced the luciferase reporter activity driven by the site $\mathrm{A}$ and site $\mathrm{B}$, but not the negative control fragment $\mathrm{C}$ (Fig. 5c). Collectively, these data demonstrated that Zeb1 repressed Pak3 transcription through binding site $\mathrm{A}$ and site $\mathrm{B}$ situated in the Pak3 promoter. To further pinpoint the binding site of Zeb1 in Pak3 promoter, we introduced point mutations to site $\mathrm{A}$ and site $\mathrm{B}$, respectively. Mutation in site A or site B alone was able to compromise the repression effect of Zeb1 partially while compound mutations in $\mathrm{A}$ and $\mathrm{B}$ compromised the effect completely, suggesting both site A and site B are critically important binding sites for Zeb1 to regulate Pak3 expression (Fig. 5d). To further corroborate our observations, we designed two pairs of primers which flanked the putative Zeb1-binding site in the Pak3 promoter for chromatin immunoprecipitation (ChIP). As shown in Fig. 5e, an obvious enrichment of Zeb1 in Pak3-binding site A and site B was detected, demonstrating a binding of Zeb1 to the promoter of Pak3 and supporting the notion that Zeb1 functioned as a transcriptional repressor of Pak3. Additionally, electroporation of an expression construct of Pak3 that was followed by an IRES-GFP cassette into the VZ of wild-type embryos at E14.5, resulted in aberrant migration of cortical neurons similar to those observed upon interference of Zeb1. Furthermore, as switching from multipolar to bipolar morphology is a critical step during radial migration and specification of neuronal subtype identity and cortical lamination, we examined the proportion of bipolar morphological cells and found that it was significantly increased upon overexpression of Pak3 compared with the controls (Fig. 5f, g). Thus, Pak3 is a critical downstream target of Zeb1 to regulate cortical neuronal migration and morphological change. 
a
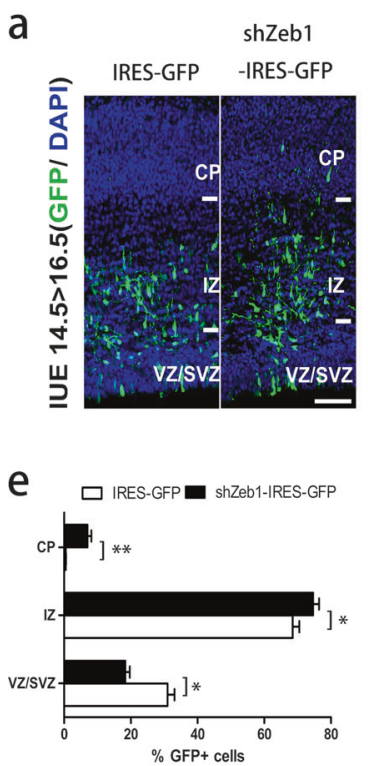

b

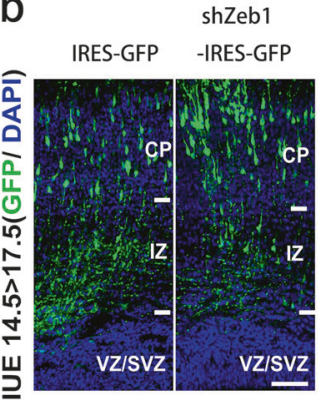

f

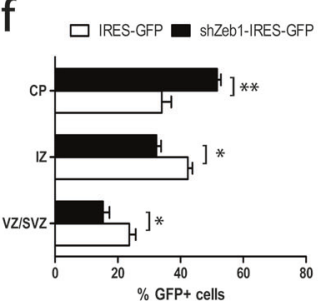

Fig. 4 Interference of Zeb1 accelerates migration of upper-layer cortical neurons. a-d Wild-type brains were electroporated at E14.5 with the plasmids of IRES-GFP and shZeb1-IRES-GFP and analyzed at (a) E16.5, (b) E17.5, (c) E18.5, and (d) P2. Nuclei were counterstained with DAPI (blue). More shZeb1-IRES-GFP-positive cells were located in the cortical plate (CP), while fewer cells were found in the

\section{Zeb1 represses Pak3 expression through recruiting PRMT5}

Zeb1 is reported to exert functions by recruiting HDAC1 and HDAC2 in pancreatic cancer [24], suggesting that transcript factors regulate gene expression with the assistance of epigenetic partners. To ask whether some coeffectors, especially epigenetic partners, participate in the processes of Zeb1 modulating Pak3 expression, we overexpressed Zeb1 in HEK293 cells and performed immunoprecipitation with $\mathrm{IgG}$ and the Zeb1 antibody. Coomassie brilliant blue staining showed that some unknown bands were precipitated by the Zeb1 antibody (Fig. 6a). To decipher this, we performed mass spectrum analysis. Interestingly, protein arginine methyltransferase 5 (PRMT5) was identified (Fig. 6b), which covalently modifies both histone and non-histone proteins, thereby suppressing the transcription as well as chromatin regulation. Furthermore, to confirm the interaction between Zeb1 and PRMT5, immunoprecipitation was performed, and formation of a complex by Zeb1 and PRMT5 could be detected by using lysates from HEK293 cells expressing GFP-Zeb1 and FLAGPRMT5 (Fig. 6c). Concomitantly, immunocytochemistry studies in N2a cells revealed that Zeb1 and PRMT5 apparently colocalized in the nucleus (Fig. 6d). Thus, we validated the interaction of Zeb1 and PRMT5. Next, we explored the orchestral functions of Zeb1 and PRMT5 in the processes of regulating of Pak3 expression. We performed
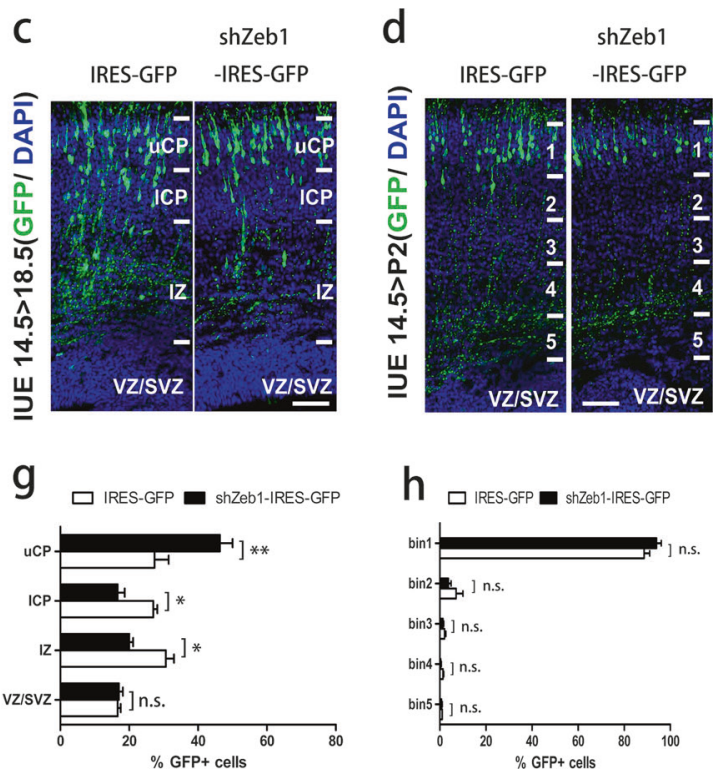

intermediate zone (IZ) and ventricular/subventricular zones (VZ/SVZ) compared with controls at E18.5. $\mathbf{e}-\mathbf{h}$ Quantification of the relative distribution of GFP-positive cells in CP, IZ, VZ/SVZ as indicated in $(\mathbf{a}-\mathbf{c})$ or five bins in $(\mathbf{d})$. All bar graphs represent mean $\pm \mathrm{SEM}$; Student's $t$ test; ns, not significant; $* p<0.05$; $* * p 0.01$; Scale bars: $50 \mu \mathrm{m}$

qPCR to detect the effect of PRMT5 on Pak3 transcription in N2a cells. Notably, the endogenous expression of Pak3 was upregulated 2 days after introduction of a PRMT5 inhibitor, EPZ015666 (Fig. 6e). Concurrently, Pak3 promoter luciferase activity was robustly upregulated at the same time point (Fig. 6f). These data demonstrated that PRMT5 suppressed Pak3 transcription. However, questions were raised as how Zeb1 and PRMT5 collaborated with each other and which of them played a leading role in this process. To determine that, luciferase assay and ChIP were performed. We noticed that suppression of Pak3 transcription by Zeb1 could be partially rescued by the PRMT5 inhibitor EPZ015666, but the suppression effect was retained compared with the vector group (Fig. 6g). Furthermore, ChIP assay showed that the enrichment of PRMT5 was reduced once Zeb1 was interfered (Fig. 6h). Considering together, Zeb1 critically suppresses Pak3 transcription with the assistance of PRMT5.

\section{Knockdown of Pak3 rescues aberrant migration of upper-layer cortical neurons in Zeb1 knockdown cortex}

To elucidate the functional relationship between Zeb1 and Pak3, we performed rescue experiments by electroporating two Pak3 short hairpins RNA plasmids into Zeb1 knockdown cells (Fig. 7). Compared with scramble shRNA, both constructs interfered Pak3 expression effectively in 

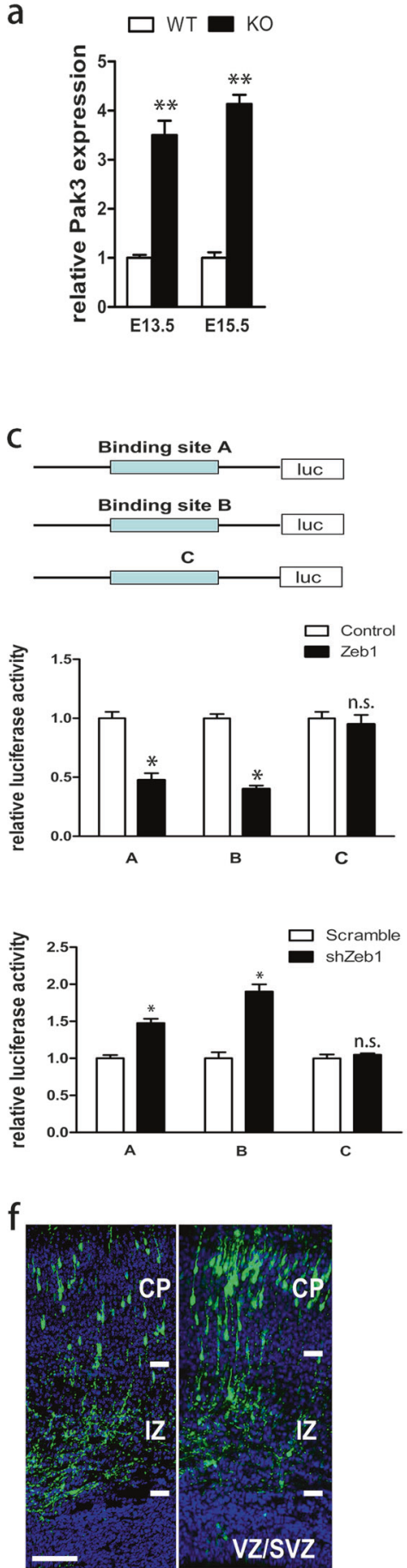

b

Binding site A Binding site B
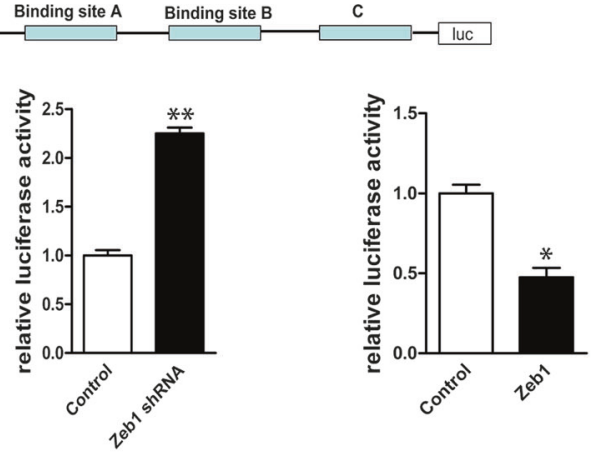

d
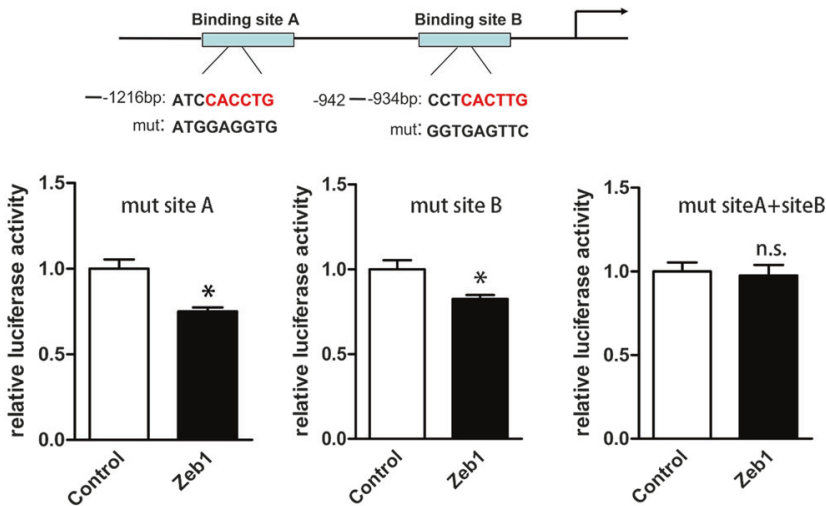

e
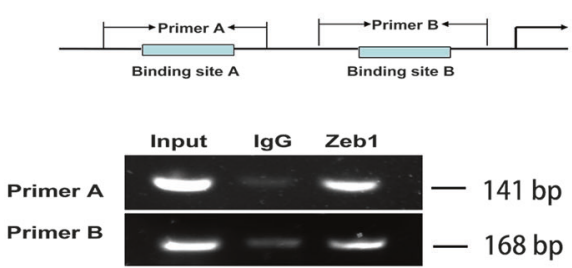

g
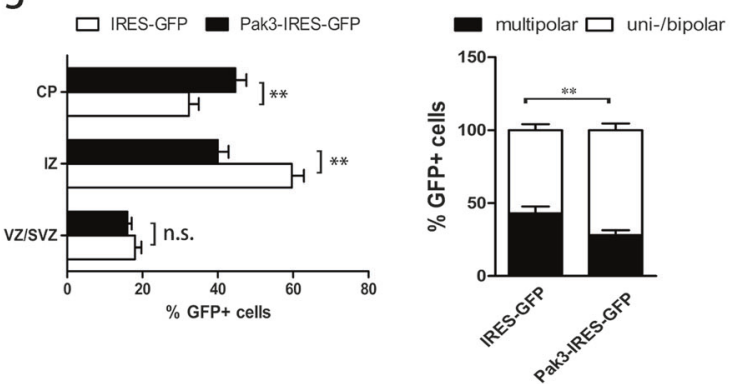

HEK293 cells (Fig. S5). Each plasmid was coelectroporated with shZeb1-IRES-GFP into the VZ of embryos at E14.5. Obviously, interference of Pak3 by shPak3-1 and shPak3-2 completely rescued aberrant neuronal migration and morphological defects in Zeb1 knockdown cortical neurons. To further confirm the phenotype of Pak3 interference, we electroporated shPak3-1 or shPak3-2, together with a knockdown-resistant Pak3* construct into Zeb1 knockdown cells. By doing this, we can restore the Zeb1-silencing phenotype, supporting the idea that Pak3 is a critical functional downstream effector of Zeb1.

\section{Discussion}

In this study, we have provided direct genetic evidence that the transcription factor Zeb1 is a pivotal factor in regulating neuronal progenitor proliferation and differentiation as well 
Fig. 5 Zeb1 acts as a transcriptional suppressor for Pak3. a Relative Pak3 mRNA expression levels determined by quantitative PCR in cortical tissue of wild-type and Zeb1 knockout mice were increased at E13.5 $(n=3)$. b Activities of site A and site B of Pak3 promoter were modulated by Zeb1. The Zeb1 regulatory site A, site B and negative control of fragment $\mathrm{C}$ were fused to luciferase reporter together. The luciferase reporter constructs were transfected with the CMV-Renilla plasmid as a internal control into HEK293 cells. Interference of Zeb1 resulted in increased luciferase activity. Overexpress Zeb1 reduced the luciferase activity ( $n=3$ in these experiments). $\mathbf{c}$ The Zeb1 regulatory site $\mathrm{A}$, site $\mathrm{B}$, and negative control of fragment $\mathrm{C}$ were fused to luciferase reporter separately and transfected with the CMV-Renilla plasmid together as a internal control into HEK293 cells. Interference of Zeb1 resulted in increased luciferase activity of site A, site B separately and no significant change in negative control of fragment $\mathrm{C}$. Overexpression of Zeb1 displayed opposite effect $(n=4$ in these experiments). d The putative of Zeb1-binding sequence A, B (red letter) were mutated in luciferase reporter constructs. e Semiquantitative analysis of Zeb1 binding to Pak3 by the ChIP assay in $\mathrm{N}_{2} \mathrm{a}$ cells. Primer A and B flanking potential Zeb1-binding sites are designed in binding site A or binding site B. Anti-Zeb1 antibody was used for the immunoprecipitation. f Electroporation of Pak3-IRESGFP into WT brains at E14.5 increased the proportion of GFP-positive cells in IZ and cortical plate (CP) at the expense of GFP-positive cells in the VZ/SVZ compared with a control vector at E17.5. Sections were counterstained with DAPI. g Quantification of the relative distribution of GFP-positive cells in CP, IZ, VZ/SVZ and morphological changes as shown in (f). All bar graphs represent mean \pm SEM; Student's $t$ test; $\mathrm{ns}$, not significant; ${ }^{*} p<0.05 ;{ }^{* *} p<0.01$; ns, not significant; Scale bars: $50 \mu \mathrm{m}$

as neuronal migration in the mouse neocortex. When Zeb1 is deleted at the onset of neurogenesis, the neuronal progenitors exit cell cycle precociously and the spindle orientation becomes tilted, leading to premature differentiation of cortical progenitors into neurons. Deficiency of Zeb1 results in reduction of the RGC pool and severe defects in the cortical layer and brain cytoarchitecture. Much more upperlayer cortical neurons are produced due to aberrant neuronal migration to their final position of superficial cortical layers. Thus, our data demonstrate that Zeb1 plays an important role in embryonic neurogenesis by maintaining a proper self-renewal progenitor pool and preventing aberrant postmitotic neuronal migration in the developing mouse cortex.

Our cell division mode analysis regarding how Zeb1 knockout affects the shift from symmetrical divisions to asymmetrical divisions of the RGCs provide a cellular mechanism for their precocious differentiation. During the transition of proliferation to differentiation, the cleavage plane of mitotic progenitors changes gradually. While most mitotic cleavage planes are vertical to the epithelial surface at E10.5, a number of the planes exhibit an oblique orientation at E14.5 [25]. The gradual orientation transition of cleavage plane is critical to allow a gradual increase in neurogenesis rates and proper cortical morphogenesis and lamination [26]. Upon removal of Zeb1, the number of progenitors with the oblique orientation of cleavage plane is significantly increased. As a larger number of BPs are generated at the expense of the progenitor pool, the tilted cleavage-plane orientation appears to be responsible for the reduction of the RGC pool. Thus, there is a shift from symmetrical to asymmetrical divisions, affecting fate specification of RGC progenies by influencing the cleavage plane of mitotic RGCs.

The present data are consistent with the notion that birth date of neurons is correlated with distinct laminar fates in the cerebral cortex [27, 28]. As Zeb1 influences spindle orientation that leads to premature neuronal differentiation, it eventually causes aberrant cortical lamination during neurogenesis. Following knockdown of Zeb1, RGCs prematurely shift from a neural stemness state to a proneuronal differentiation state and differentiated into cortical neurons ahead of the normal developmental schedule (Fig S6). These data suggest that knockdown of Zeb1 disturbs the temporal competence of cortical neural stem cells, thereby perturbing the timing of neuronal birth. Furthermore, in the present study, deletion of Zeb1 perturbs the thickness of cortical layer severely. In particular, the upper cortical layer marked by Brn2 is increased (Fig. 2a). Conversely, the deep-layer neurons respond to the deficiency of Zeb1 variably. While the layer marked by Ctip2 is barely changed, the layer labeled by Tbr1 is significantly reduced (Fig. 2c-f). Support for this outcome comes from our detailed spatiotemporal assessment of Zeb1 expression, showing that Zeb1 is expressed in the Pax6-positive RGCs rather than Tbr2-positive basal progenitors. The data suggest that Zeb1 controls a lineage of RGCs population, but not basal progenitors. Thus, Zeb1 is likely to act selectively on the actively dividing RGCs that produce upper-layer neurons. The aberrant neuronal migration and extra number of upper-layer neurons is attributable to the initial precocious differentiation of RGCs.

An important finding in our study is to show that Zeb1 binds to the promoter of Pak3 that is a novel and an unreported downstream target of Zeb1 and exerts its function through direct repression of Pak3 transcription, concomitantly, by recruiting PRMT5 to enhance the repression effect. Overexpression of Pak3 in wild-type cortical neurons recapitulates the Zeb1-mutant phenotype, whereas knockdown of Pak3 in Zeb1-deficient cortical neurons rescues it. In this process, we identify a suppressant PRMT5, binding to and being recruited by Zeb1, which reinforces the notion that Zeb1 represses the transcription of Pak3. Pak3 is one member of the p21-activated kinase (PAKs) family and serves as important regulators of cytoskeletal dynamics and cell motility, transcription activities, and cell-cycle progression $[29,30]$.

Taken together, the present data show that Zeb1 plays an essential role in maintaining a proper size of the progenitor pool. Depletion of Zeb1 disturbs the balance and rhythm between the proliferation and differentiation of RGCs, 
Fig. 6 Zeb1 recruits PRMT5 to suppress Pak3 transcription. a, b Coomassie brilliant blue staining and identification of Zeb1binding protein by liquid chromatography-tandem mass spectrometry: the PRMT5 peptides identified through mass spectrometry are shown. c Interaction of Zeb1 with PRMT5 in HEK293 cells. Lysates from HEK293 cells transiently expressing FLAG-PRMT5 and/ or EGFP-Zeb1 were immunoprecipitated (IP) with antibody against Zeb1 and analyzed by immunoblotting with antibodies against FLAG and Zeb1. d Endogenous Zeb1 and PRMT5 in N2a cells were visualized using

immunofluorescence

microscopy. Nuclei were stained with DAPI; scale bar: $10 \mu \mathrm{m}$. e, f Increased endogenous Pak3 mRNA level and Pak3 promoter luciferase activity in N2a cells 2 days after PRMT5 inhibitor EPZ015666 $(5 \mu \mathrm{M})$ was

administered. g Suppression of Pak3 luciferase activity by Zeb1 could be partially rescued by PRMT5 inhibitor EPZ015666. h PRMT5 enrichment on Pak3 promoter could be compromised by interference of Zeb1 in N2a cells a IgG IP-Zeb1

b

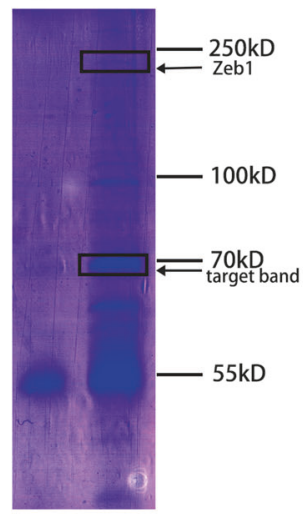

AAILPTSIFLTNK charge $=2 \quad M / Z=694.91$

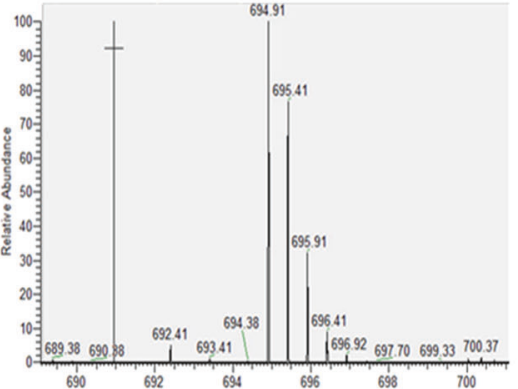

d
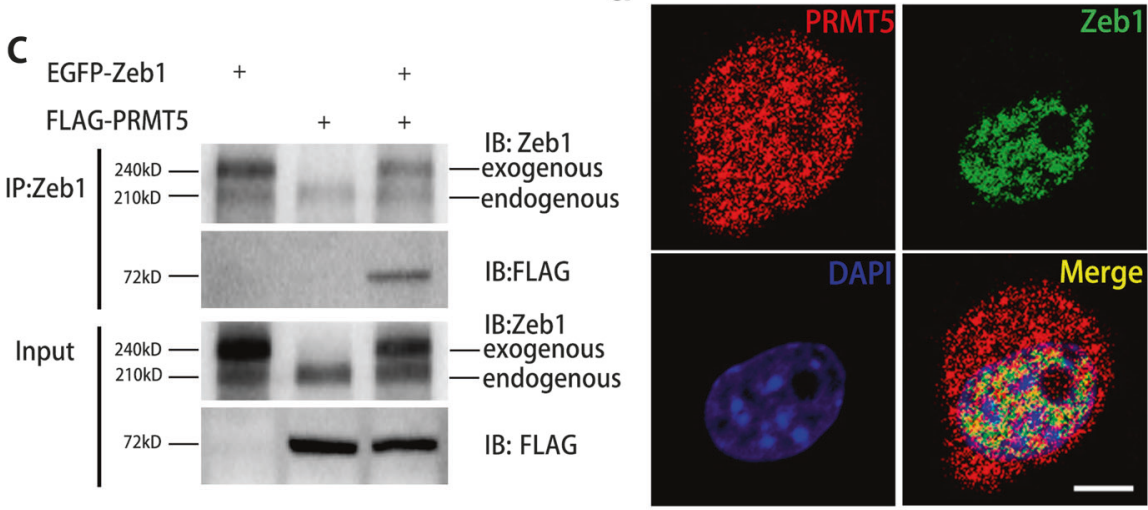

e

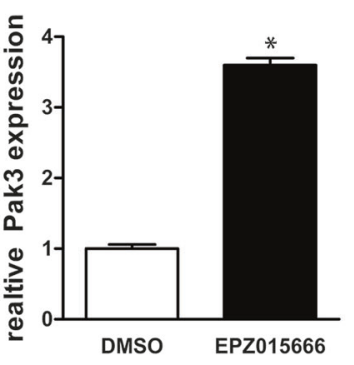

f

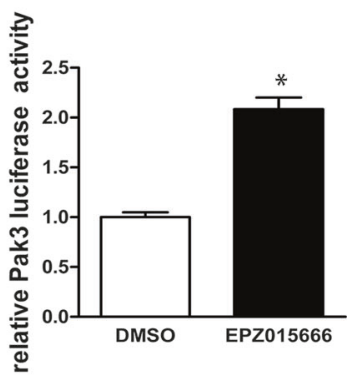

g

h

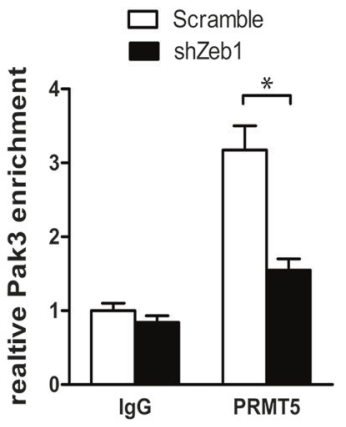


a
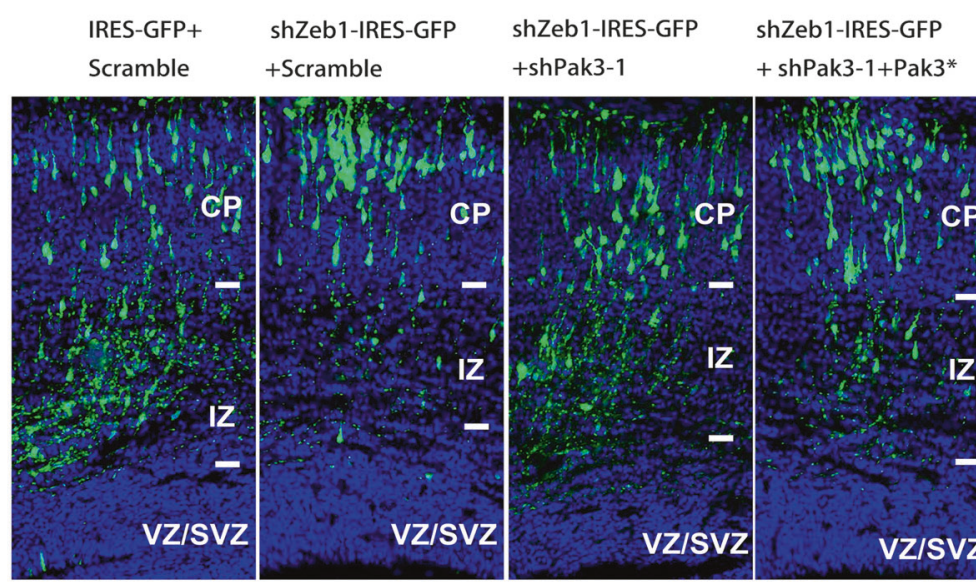

shZeb1-IRES-GFP

shZeb1-IRES-GFP

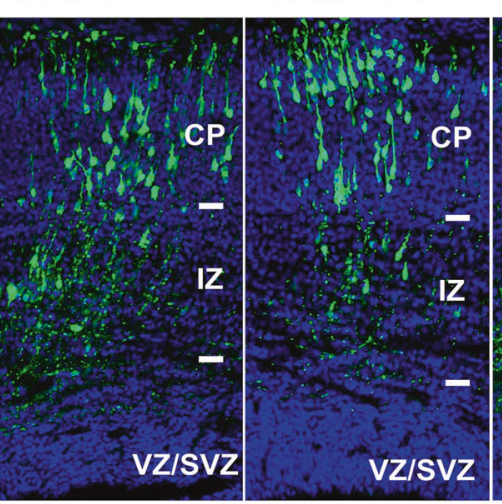

+ shPak-2

+ shPak3-2+Pak3*

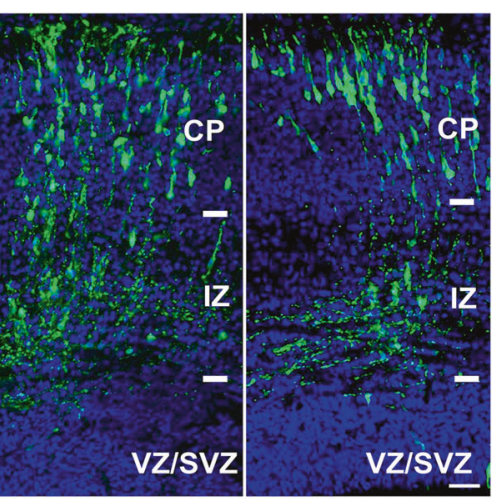

b

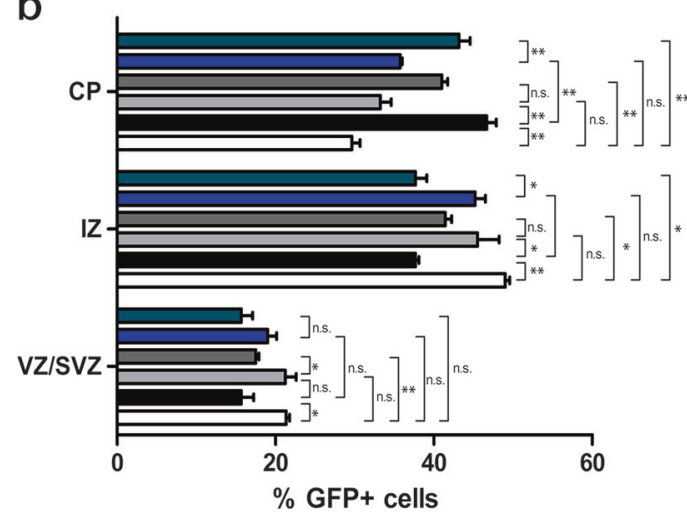

Fig. 7 Knockdown of Pak3 in Zeb1-silenced upper-layer cortical neurons rescues polarization and aberrant migration. a Coelectroporation of shZeb1-IRES-GFP and Scramble shRNA into wild-type brains at E14.5 increased the proportion of GFP-positive cells in the cortical plate (CP) at the expense of GFP-positive cells in the ventricular/subventricular zone (VZ/SVZ) and intermediate zone (IZ) at E17.5 compared with IRES-GFP, together with a Scramble shRNA. Co-electroporation of shZeb1-IRES-GFP with shPak3-1 and shPak3-2 separately rescued the phenotype. Co-electroporation of shZeb1-IRES-GFP, shPak3-1 or shPak3-2, and an interferential-

resulting in RGCs differentiating earlier, producing a large number of premature upper-layer neurons that will migrate away rapidly. Importantly, our study also identifies for the first time that Pak3 is a novel target of Zeb1. The function of Zeb1 may provide insights into the molecular mechanisms underlying developmental diseases in the brain.

\section{Materials and methods}

\section{Mouse models}

All experimental mice were housed in animal center, which is pathogen-free and with controlled temperature and humidity at Renji Hospital of Shanghai Jiao Tong University. Zeb1 knockout mice were purchased from RIKEN

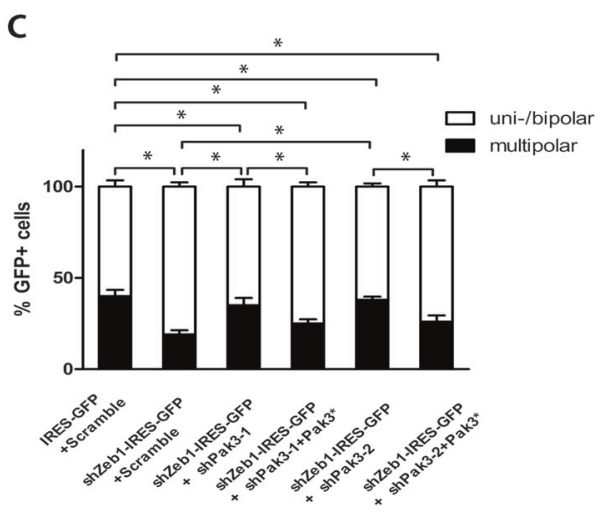

resistant Pak3 construct (Pak3*) could mimic the phenotype. Nuclei were stained with DAPI. b Quantification of experiments shown in (a) ( $n=3$ for each group). $\mathbf{c}$ The proportions of bipolar and multipolar GFP-positive cells in the E17.5 cortex electroporated as indicated at E14.5 $(n=3)$. Co-electroporation of shZeb1-IRES-GFP, together with Pak3 scramble shRNA, increased the proportion of bipolar at the expense of multipolar cells compared to IRES-GFP, together with a Pak3 Scramble shRNA. Co-electroporation of shZeb1-IRES-GFP and shPak3-1 or shPak3-2 rescues the phenotype

BRC (RBRC01923). All animal experimental protocols were according with the instruction of Animal Research Ethics Committee of Renji Hospital.

\section{Immunoblotting}

For western blot analysis, samples were lysed in RIPA buffer (\#89900, Pierce, Waltham, MA, USA) supplemented with the protease inhibitor cocktail (\#78429, Thermo scientific, Waltham, MA, USA) and then were separated by SDS-PAGE gels and transferred to nitrocellulose membranes by electroblotting. Membranes were preincubated in $5 \%$ nonfat milk in Tris-buffered solution and $0.1 \%$ Tween20 (TBST) for $2 \mathrm{~h}$, then incubated with primary antibody overnight at $4{ }^{\circ} \mathrm{C}$ and washed three times in TBS, then incubated with horseradish peroxidase-conjugated 
secondary antibody for $1.5 \mathrm{~h}$, and developed using the SuperSignal West PicoChemiluminescent Substrate (\#34580, Pierce). Antibodies were used as follows: Zeb1 (\#21544-1-AP, Proteintech, Rosemont, IL, USA). $\beta$-actin (\#A5441, Sigma Aldrich) was used for internal loading control.

\section{Quantitative PCR}

The total RNA was extracted using TRIzol Kit following by the manufacturer's instructions. The cDNA syntheses were performed using PrimeScript RT Kit (\#RR037A, Takara, Dalian, China). Real-time PCR Super Mix (\#QPK-201, Toyobo, Osaka, Japan) was used for quantitative PCR. Quantitative PCR was performed on the Step one Plus RTPCR Systems (Applied Biosystems, MA, USA). The primers for quantitative PCR were as follows.

\begin{tabular}{lll} 
Gene & Forward & Reverse \\
actin & GGCTGTATTC & CCAGTTG \\
& CCCTCCATCG & GTAACAA \\
& & TGCCATGT \\
Pak3 & TTGGATAACG & GGGCACA \\
& AAGAAAAACC & TCTGTGA \\
& CCC & GCCATAG \\
\hline
\end{tabular}

\section{In utero electroporation}

In utero electroporations were performed as described previously. Briefly, at E14.5, pregnant mice were anesthetized and uterine horns were exposed, then $1.5 \mu \mathrm{g} / \mu \mathrm{l}$ plasmids solutions were injected into the lateral ventricle. Platinum electrodes ( $5 \mathrm{~mm}$, NEPA GENE) were positioned on both side of the embryonic head and five $50 \mathrm{~ms}$ pulses of $30 \mathrm{mV}$ with $950 \mathrm{~ms}$ intervals were applied with an electroporator (NEPA GENE, CUY21 EDIDT). After that, uterine horns were placed back to the abdominal cavity, and wounds were sutured. All procedures were approved by the institutional animal care.

\section{Constructs}

The full-length coding sequence of Zeb1 (Genbank: NM_011546.3) was cloned into PCDNA3.1 fusion with GFP or FLAG to generate PCDNA3.1-Zeb1-GFP and PCDNA3.1-Zeb1-FLAG and the coding sequence of Pak3 (Genbank: NM_001195046.1) was cloned by PCR and inserted into pCIG to generate pCIG-Pak3-IRES-GFP and pCIG-Pak3-FLAG. The coding sequence of PRMT5 (Genbank: NM_013768.3) was cloned into PCDNA3.1 fusion with FLAG to generate pCDNA3.1-PRMT5-FLAG. shPak3-1 and shPak3-2 were generated by cloning $5^{\prime}$ TAGCAGCACATCAGTCGAATA-3' and 5'-CCCAATAT TGTCAATTATTTA- ${ }^{\prime}$, respectively, into the short hairpin RNA vector, pSUPER- NEO. shZeb1-1 and shZeb1-2 were generated by cloning 5'-GTCGACAGTCAGTAGCG TTTA-3' and 5'-ATAGAGGCTACAAGCGCTTTA-3', respectively, into the short hairpin RNA vector, pSUPEREGFP. The sequence of knockdown-resistant Pak3 expression construct (pCIG-Pak3*) harboring silent point mutations recognized by Pak3 shRNA\#1 and \#2 were as follows: 5'-TA GCC GCC CAC CAG $\underline{A G C}$ AAC A-3' and 5'-CCC AAC AT므 GTG A $\underline{A} \underline{C}$ TA $\underline{C}$ CTG-3'. The underlined nucleotides identify silent mutations of nt564 $(\mathrm{A} \rightarrow \mathrm{C})$, $\mathrm{nt567}(\mathrm{A} \rightarrow \mathrm{C}), \mathrm{nt569}(\mathrm{T} \rightarrow \mathrm{C}), \mathrm{nt573}(\mathrm{T} \rightarrow \mathrm{A}), \mathrm{nt574}(\mathrm{C} \rightarrow$ $\mathrm{G}), \mathrm{nt575}(\mathrm{G} \rightarrow \mathrm{C}), \mathrm{nt578}(\mathrm{T} \rightarrow \mathrm{C}), \mathrm{nt1083}(\mathrm{T} \rightarrow \mathrm{C}), \mathrm{nt1086}$ $(\mathrm{C} \rightarrow \mathrm{G}), \mathrm{nt} 1089(\mathrm{~T} \rightarrow \mathrm{C}), \mathrm{nt1092}(\mathrm{T} \rightarrow \mathrm{C})$, nt1093 $(\mathrm{T} \rightarrow \mathrm{C})$, nt1095 $(\mathrm{A} \rightarrow \mathrm{G})$ of the Pak3-coding sequence.

\section{Immunohistochemistry}

Mouse brains were fixed in $4 \%$ PFA for $2 \mathrm{~h}$ at $4{ }^{\circ} \mathrm{C}$, then incubated overnight at $4{ }^{\circ} \mathrm{C}$ and followed by cryoprotection in OCT (Sakura Finetech). Coronal sections were prepared with a cryostat to yield $12-\mu \mathrm{m}$-thick coronal sections (Leica Micro-systems). Brain sections were permeabilized using $0.3 \%$ Triton $\mathrm{X}-100$ for $20 \mathrm{~min}$, then incubated with blocking solution $(0.3 \%$ Triton $\mathrm{X}-100$, $10 \%$ normal goat serum) for $2 \mathrm{~h}$ at room temperature, then incubated with primary antibody overnight at $4{ }^{\circ} \mathrm{C}$. The antibodies have been used as follows: Zeb1 (1:200, \#sc10572, Santa Cruz,), Nestin (1:200, \#N5413, Sigma Aldrich), PH3 (1:300, \#9701, Cell Signaling), Pax6 (1:300, \#ab5790, Abcam), caspase-3 (1:300, \#13847, Cell Signaling), GFP (1:1,000, \#ab13970, Abcam), goat antiBrn2 (1:200, \#sc6029, Santa Cruz,), Tbr1 (1:200, \#ab31940, Abcam), Sox2 (1:200, \#ab97959, Abcam), ZO-1 (1:100, \#ab190085, Abcam). After incubation with the primary antibody, sections were washed three times in PBS and incubated with secondary antibodies conjugated to Alexa Fluor dyes for $1 \mathrm{~h}$ at room temperature before capture.

\section{Luciferase assays}

Zeb1 plasmid and Pak3 luciferase construct were transfected together with Renilla construct using Lipofectamine 3000 Transfection Reagents (\#L3000001, Life). Twelve hours later, fresh media with the addition of PRMT5 inhibitor EPZ015666 (SELLECK) was added, and $48 \mathrm{~h}$ later, cells were lysed and analyzed with the Dual-Luciferase Reporter Assay System (\#E1910, Promega, Madison, WI, USA). Luciferase activity values were normalized to the 
Renilla value. The primers using for luciferase plasmids were as follows:

\begin{tabular}{ll} 
mut-site A & Forward: CAAGAGATGGAGGTGTCT \\
& CTGCCTCTCCAGTGCTGG \\
& Reverse: GCAGAGACACCTCCATCT \\
& CTTGAATTCAAGGCCAGCC \\
mut-site B & Forward: ACTTTTAGAAGGTGAGTTC \\
& TGCCTGTGAAAGTCAAC \\
& Reverse: TCACAGGCAGAACTCACCTTC \\
& TAAAAGTGTCTTTG \\
\hline
\end{tabular}

\section{Immunoprecipitation}

Zeb1 and Pak3 plasmids were transfected into HEK293 cells and $48 \mathrm{~h}$ later cells were homogenized with lysis buffer (5 mM EDTA, $10 \mathrm{mM}$ Tris [pH 7.5], 0.5\% Triton X-100, $135 \mathrm{mM} \mathrm{NaCl}$ ) containing protease inhibitor and protein phosphatase inhibitor (\#78429, Thermo scientific, Waltham, MA, USA). The lysates were subjected to immunoprecipitation with IgG or Zeb1 antibody by protein A/G (\#SC2003, Santa Cruz, TX, USA).

\section{Cell-cycle exit assay}

EdU labeling was performed by intraperitoneal injection of $100 \mathrm{mM}$ of $1 \mathrm{mg} / \mathrm{ml}$ EdU in DMSO into pregnant mice embryos at E14.5. Mice were killed $24 \mathrm{~h}$ later, and embryonic brains were dissected and sectioned. Sections were stained with anti-Ki67 antibody (\#ab15580, Abcam) and post fixed with 4\% PFA before the EdU (\#900584, Sigma Aldrich) detection.

\section{Pair cell analysis}

The embryonic brains were dissected at E14.5 and the cortex were isolated, incubated in a protease solution containing 10 units/ml papain (\#P4762, Sigma Aldrich) for 10 min, and then triturated to create a single-cell suspension. Cells were washed three times with the culture medium containing DMEM and plated onto coverslips coated with poly-L-lysine (\#P4707, Sigma Aldrich) in 24-well plates at clonal density. The staining was performed $24 \mathrm{~h}$ later.

\section{ChIP assay}

The N2A cells were transfected with pSUPER-shZeb1 plasmid. Forty-eight hours later, cells were harvested and fixed with 1\% PFA. ChIP assay was performed using ChIP Kit (\#9003, CST) according to the manufacturer's instruction. Antibody against Zeb1 (\#21544-1-AP, Proteintech) was used to immunoprecipitate the Zeb1-DNA complex and antibody against PRMT5 (\#GTX116004, GenTex) was used to immunoprecipitate the PRMT5-DNA complex. Quantitative PCR was performed to assess the enrichment of Zeb1 in indicated Pak3-regulatory elements. The primers for PCR amplification were as follows:

\begin{tabular}{lll}
\hline & Forward & Reverse \\
Pak3-site A & TGACAGAGTT & TCTTCTGCTAGGTGGT \\
& CCTCTGCATAC & GGTAG \\
Pak3-site B & ATGAAAACCA & TGAATTTCTCTGTAAC \\
& CGAATTCATTC & TTAGG \\
\hline
\end{tabular}

\section{Sorting of GFP-positive cells and qPCR}

At embryonic day 10.5 (E10.5), $1.5 \mu \mathrm{l}$ shZeb1-IRES-GFP or IRES-GFP retrovirus were injected into the later ventricle. Thirty hours later, the GFP-positive cells were isolated from the cortex by flow cytometry. Then, the relative expression of Nestin, Sox2, Pax6, and Ascl1 were analyzed by qPCR and the primers are as follows:

\begin{tabular}{lll} 
Gene & Forward & Reverse \\
actin & GGCTGTATTCC & CCAGTTGGTAACAATGC \\
& CTCCATCG & CATGT \\
Sox2 & CATCCACTTCTA & AGCTCCCTGTCAGGTCC \\
& CCCCACCTT & TT \\
Nestin & CCCTGAAGTCGA & CTGCTGCACCTCTAAGC \\
& GGAGCTG & GA \\
Ascll & GCAACCGGGTCA & GTCGTTGGAGTAGTTGG \\
& AGTTGGT & GGG \\
Pax6 & TACCAGTGTCTAC & TGCACGAGTATGAGGAG \\
& CAGCCAAT & GTCT \\
\hline
\end{tabular}

Acknowledgements This study was supported by funds from Ministry of Science and Technology of the People's Republic of China (2017YFA0102900 to WQG), National Natural Science Foundation of China (81872406, 81630073 to WQG and 81773115 to WX), Science and Technology Commission of Shanghai Municipality (16JC1405700 to WQG), High Peak IV fund from Education Commission of Shanghai Municipality on Stem Cell Research (to WQG) and KC Wong foundation (to WQG) and SJTU interdisciplinary grant (YG2017MS52 to WX).

\section{Compliance with ethical standards}

Conflict of interest The authors declare that they have no conflict of interest.

Publisher's note: Springer Nature remains neutral with regard to jurisdictional claims in published maps and institutional affiliations. 


\section{References}

1. Johansson PA, Cappello S, Gotz M. Stem cells niches during development--lessons from the cerebral cortex. Curr Opin Neurobiol. 2010;20:400-7.

2. Popovitchenko T, Rasin MR. Transcriptional and posttranscriptional mechanisms of the development of neocortical lamination. Front Neuroanat. 2017;11:102.

3. Gotz M, Barde YA. Radial glial cells defined and major intermediates between embryonic stem cells and CNS neurons. Neuron. 2005;46:369-72.

4. Stancik EK, Navarro-Quiroga I, Sellke R, Haydar TF. Heterogeneity in ventricular zone neural precursors contributes to neuronal fate diversity in the postnatal neocortex. J Neurosci. 2010;30:7028-36.

5. Attardo A, Calegari F, Haubensak W, Wilsch-Brauninger M, Huttner WB. Live imaging at the onset of cortical neurogenesis reveals differential appearance of the neuronal phenotype in apical versus basal progenitor progeny. PLoS ONE. 2008;3:e2388.

6. Kosodo Y, Roper K, Haubensak W, Marzesco AM, Corbeil D, Huttner WB. Asymmetric distribution of the apical plasma membrane during neurogenic divisions of mammalian neuroepithelial cells. EMBO J. 2004;23:2314-24.

7. Mapelli M, Gonzalez C. On the inscrutable role of Inscuteable: structural basis and functional implications for the competitive binding of NuMA and Inscuteable to LGN. Open Biol. 2012;2:120102.

8. Chou FS, Li R, Wang PS. Molecular components and polarity of radial glial cells during cerebral cortex development. Cell Mol Life Sci. 2018;75:1027-41.

9. McManus MF, Golden JA. Neuronal migration in developmental disorders. J Child Neurol. 2005;20:280-6.

10. Jheng GW, Hur SS, Chang CM, Wu CC, Cheng JS, Lee HH, et al. Lis1 dysfunction leads to traction force reduction and cytoskeletal disorganization during cell migration. Biochem Biophys Res Commun. 2018;497:869-75.

11. Wegiel J, Kuchna I, Nowicki K, Imaki H, Wegiel J, Marchi E, et al. The neuropathology of autism: defects of neurogenesis and neuronal migration, and dysplastic changes. Acta Neuropathol. 2010;119:755-70.

12. Williams ML, Solnica-Krezel L. Regulation of gastrulation movements by emergent cell and tissue interactions. Curr Opin Cell Biol. 2017;48:33-9.

13. Nikolopoulou E, Galea GL, Rolo A, Greene ND, Copp AJ. Neural tube closure: cellular, molecular and biomechanical mechanisms. Development. 2017;144:552-66.

14. Brabletz T, Kalluri R, Nieto MA, Weinberg RA. EMT in cancer. Nat Rev Cancer. 2018;18:128-34.

15. Zhang P, Sun Y, Ma L. ZEB1: at the crossroads of epithelialmesenchymal transition, metastasis and therapy resistance. Cell Cycle. 2015;14:481-7.
16. Takagi $\mathrm{T}$, Moribe $\mathrm{H}$, Kondoh $\mathrm{H}$, Higashi $\mathrm{Y}$. DeltaEF1, a zinc finger and homeodomain transcription factor, is required for skeleton patterning in multiple lineages. Development. 1998;125:21-31.

17. Higashi Y, Moribe H, Takagi T, Sekido R, Kawakami K, Kikutani $\mathrm{H}$, et al. Impairment of $\mathrm{T}$ cell development in deltaEF1 mutant mice. J Exp Med. 1997;185:1467-79.

18. Siles L, Sanchez-Tillo E, Lim JW, Darling DS, Kroll KL, Postigo A. ZEB1 imposes a temporary stage-dependent inhibition of muscle gene expression and differentiation via CtBP-mediated transcriptional repression. Mol Cell Biol. 2013;33:1368-82.

19. Wang X, Tsai JW, LaMonica B, Kriegstein AR. A new subtype of progenitor cell in the mouse embryonic neocortex. Nat Neurosci. 2011;14:555-61.

20. Uzquiano A, Gladwyn-Ng I, Nguyen L, Reiner O, Gotz M, Matsuzaki F, et al. Cortical progenitor biology: key features mediating proliferation versus differentiation. J Neurochem. 2018;146:500-25.

21. Govindan S, Jabaudon D. Coupling progenitor and neuronal diversity in the developing neocortex. FEBS Lett. 2017;591:3960-77.

22. Namba T, Huttner WB. Neural progenitor cells and their role in the development and evolutionary expansion of the neocortex. Wiley Interdiscip Rev Dev Biol. 2017; 6:216-22.

23. Souopgui J, Solter M, Pieler T. XPak3 promotes cell cycle withdrawal during primary neurogenesis in Xenopus laevis. EMBO J. 2002;21:6429-39.

24. Aghdassi A, Sendler M, Guenther A, Mayerle J, Behn CO, Heidecke $\mathrm{CD}$, et al. Recruitment of histone deacetylases HDAC1 and HDAC2 by the transcriptional repressor ZEB1 downregulates Ecadherin expression in pancreatic cancer. Gut. 2012;61:439-48.

25. Postiglione MP, Juschke C, Xie Y, Haas GA, Charalambous C, Knoblich JA. Mouse inscuteable induces apical-basal spindle orientation to facilitate intermediate progenitor generation in the developing neocortex. Neuron. 2011;72:269-84.

26. Yingling J, Youn YH, Darling D, Toyo-Oka K, Pramparo T, Hirotsune $S$, et al. Neuroepithelial stem cell proliferation requires LIS1 for precise spindle orientation and symmetric division. Cell. 2008;132:474-86.

27. Mihalas AB, Elsen GE, Bedogni F, Daza RAM, Ramos-Laguna $\mathrm{KA}$, Arnold SJ, et al. Intermediate progenitor cohorts differentially generate cortical layers and require Tbr2 for timely acquisition of neuronal subtype identity. Cell Rep. 2016;16:92-105.

28. Schuurmans C, Armant O, Nieto M, Stenman JM, Britz O, Klenin $\mathrm{N}$, et al. Sequential phases of cortical specification involve neurogenin-dependent and -independent pathways. EMBO J. 2004;23:2892-902.

29. Bokoch GM. Biology of the p21-activated kinases. Annu Rev Biochem. 2003;72:743-81.

30. Rane CK, Minden A. P21 activated kinase signaling in cancer. Semin Cancer Biol. 2018;54:40-9. 